\title{
LE TRAITEMENT CHIRURGICAL DES POCHES DE RÉTRACTION TYMPANIQUES
}

\section{SURGICAL MANAGEMENT OF TYMPANIC RETRACTION POCKETS}

\author{
S. BENZARTI, A. SETHOM, S. CHNITIR, KH. RIAHI, R. BEN M'HAMMED, I. MILED, MK. CHEBBI \\ SERVICE ORL ET CHIRURGIE MAXILLO-FACIALE - HÔPITAL MILITAIRE DE TUNIS.
}

\begin{abstract}
RESUME
Les poches de rétractions représentent un aspect particulier de l'otite moyenne chronique affectant la morphologie tympanique et pouvant avoir des répercussions fonctionnelles auditives. L'évolution naturelle de l'otite atelectasique vers la maladie cholestéatomateuse complique d'avantage la prise en charge des poches de rétraction.

Les auteurs rapportent une étude rétrospective à propos de 137 cas de poches de rétraction colligées sur une période de 10 ans de 1992 à 2001.

Le traitement chirurgical des poches de rétraction pose la problématique du bon choix de la technique opératoire et de l'indication de la chirurgie de renforcement.
\end{abstract}

\section{SUMMARY}

Retraction pockets represent a particular aspect of chronic otitis whish affect tympanic membran morphology and can influence hearing potentialities. The evolution course of atelectasic disease leeding to localized retraction may be the principal cause for cholesteatoma witch complicates the management of the retraction pockets.

The authors report a retrospective study about 137cases of retraction pockets collaged from 1992 to 2001.

The problems of the surgical treatement of the retraction pockets include the best choice of the operative technique for reconstructive tynpanoplastie.

\section{INTRODUCTION}

Les poches de rétractions (PDR) se définissent comme un collapsus partiel ou total du tympan dans la caisse de l'oreille moyenne. Elle constitue une véritable maladie évolutive de la membrane tympanique dont la genèse et les mécanismes physiopathogéniques sont encore discutés.

Le génie évolutif de ces états pré-cholestéatomateux est imprévisible ce qui pose la problématique de la place et des indications de la chirurgie dans la stratégie thérapeutique face à une PDR.

\section{MATÉRIEL ET MÉTHODES}

Nous rapportons une étude rétrospective portant sur 134 poches de rétractions opérées au service d'ORL et Chirurgie Maxillo-faciale - Hôpital Militaire de Tunis entre 1992 et 2001 soit concernant 127 patients.

L'étude des dossiers nous a permis de préciser l'âge, le sexe, les signes fonctionnels et les circonstances de découverte de la maladie et de spécifier les caractéristiques de la PDR à l'examen otoscopique à savoir le siège, le stade selon la classification de Charachon ainsi que l'état de l'oreille controlatérale.

Tous nos patients ont bénéficié d'un audiogramme et d'une tympanométrie.

Nous avons évalué le Rinne préopératoire moyen sur les fréquences 500, 1000 et 2000 hz. Quatre patients ont bénéficié d'une tomodensitométrie (TDM) préopératoire. En peropératoire nous avons noté, le siège, l'extension de la poche, l'état de la muqueuse, de la chaîne ossiculaire et la présence ou non de cholestéatome. Le type d'intervention et la technique de renforcement ont été également spécifiés. Nous avons évalué nos patients par l'étude des résultats anatomiques et fonctionnels après un recul postopératoire minimum de 21 mois. Ainsi, neuf malades ont eu une TDM postopératoire et tous les patients ont bénéficié d'un examen audiométrique

\section{RESULTATS}

L'age moyen de nos patients était de 26 ans avec des extrêmes allant de 6 à 63 ans. Le sexe ratio est de 1.3. Les circonstances de découverte étaient variables. L'interrogatoire et l'étude de l'histoire clinique a révélé que 20 patients avaient un passé d'otite séromuqueuse ce qui a motivé la mise en place d'un aérateur transtympanique (ATT) au jeune âge. L'hypoacousie dominait la symptomatologie fonctionnelle (53.3\%). Vingt six pour cent des patients présentaient des épisodes otorrhéïques associées à des otalgies dans $10 \%$ des cas.

La répartition selon la classification de Charachon montre que les stades III étaient plus fréquents ( 113 cas) que les stades I (un cas) et stade II (20 cas). La PDR s'associait à une perforation dans $8 \%$ des cas. La pars tensa était concernée par le processus de rétraction dans $93 \%$ des 
cas. Le siège postérieur était le plus fréquemment retrouvé $(65 \%)$. A noter que $15 \%$ des rétractions intéressaient toute la membrane tympanique

\begin{tabular}{l|c|c|} 
& Nombres de cas & Pourcentage \\
\hline Attique antérieure & 24 & 18 \\
\hline Attique postérieure & 83 & 62 \\
\hline Hypotympanum & 4 & 3 \\
\hline Région des fenêtres & 67 & 50 \\
Sinus tympanum & 30 & 22 \\
Recessus facial & 73 & 55
\end{tabular}

Tableau I : Extension des poches de rétraction

L'examen otoscopique de l'oreille controlatérale a retrouvé un tympan pathologique dans $56 \%$ des cas, il s'agissait le plus souvent d'une poche de rétraction.

A la tympanométrie, le tracé était de type $\mathrm{C}$ dans $31 \%$ des cas, de type A dans $27 \%$ des cas et de type B dans $2 \%$ des cas. L'exploration audiométrique a révélé une audition subnormale du côté opéré dans $8 \%$ des cas correspondant tous à des PDR de stade I et II. Le rinne préopératoire moyen était évalué à $28 \mathrm{~dB}$.

Une TDM préopératoire a été indiquée chez les 4 patients aux antécédents otorrhéïques et dont la poche était de stade III. II n'a pas été objectivé de signes prédictifs de cholestéatome.

Un traitement médical associant une antibiothérapie, une corticothérapie, des mucolytiques et des topiques locaux a été institué en préopératoire à 70 patients qui présentaient des poches humides.

Tous nos patients ont été opérés, nous avons majoritairement opté pour la voie rétroauriculaire ( $97 \%$ des cas). La voie endaurale n'a été utilisée que chez 5 patients. En peropératoire, la chaîne ossiculaire était interrompue dans $47 \%$ des cas. II s'agissait d'une lyse de la branche descendante de l'enclume dans $30 \%$ des cas et de la superstructure de l'étrier dans $17 \%$ des cas.

Quinze pour cent des poches de rétractions s'associaient à une atticotomie ou à une lyse du cadre osseux. La muqueuse du fond de caisse était inflammatoire dans $27 \%$ des cas. Nous avons constaté la présence de la glue rétrotympanique chez 4 patients.

L'exploration peropératoire de la poche, elle-même, a retrouvé une extension à la région des fenêtres, au recessus facial, à l'attique postérieure respectivement dans $94 \%$, $90 \%$ et $60 \%$ des cas. Nous avons découvert fortuitement un cholestéatome associé dans $18 \%$ des cas. Chez 14\% des patients, une simple chirurgie de renforcement tympanique a été pratiquée, il s'agit des stades I et II.

Une antroatticotomie d'aération et de drainage permettant une reperméabilisation de défilé antro-addito-attrial a été pratiquée dans $68 \%$ des cas. Elle s'associait à une technique fermée avec une tympanotomie postérieure dans tous les cas. La technique ouverte avec un aménagement d'une mini-caisse n'a été faite que chez $10 \%$ des patients.

Une ossiculoplastie a été réalisée lors du premier temps chirurgical dans $28 \%$ des cas ; à noter qu'une prothèse en hydroxyapatite a été montée chez 9 malades.

Les matériaux utilisés pour le renforcement étaient de l'aponévrose temporale dans $26 \%$ des cas, du périchondre dans $10 \%$ des cas et du cartilage doublé de son périchondre dans $64 \%$ des cas. Une lame de Silastic a été interposée dans la caisse ou dans le défilé antro-attical chez $36 \%$ des malades.

Nous avons placé un ATT en fin d'intervention chez 4 patients du côté controlatéral à l'oreille opérée.

Un second look opératoire a été pratiqué dans 7 cas, il s'agissait soit de cholestéatomes découverts lors du premier temps opératoire (4cas) ou de poches de rétraction mésotympaniques postétieures (3 cas) pour lesquelles le risque de cholestéatome résiduel était important.

Après un recul minimum de 21 mois, le résultat anatomique été satisfaisant dans $88 \%$. Ailleurs l'évolution s'est faite soit vers la rétraction secondaire (10\%) ou vers la perforation (12\%). Aucune poche n'a évolué vers le cholestéatome.

L'analyse selon le siège montre que les récidives sont plus fréquentes pour les poches postérieures (10\%) que pour les poches atticales (7\%) ou globales (5\%).

L'analyse des résultats en fonction des matériaux de reconstitution montre un taux de récidive plus élevé pour les greffes d'aponévrose temporales (15\%) comparé au cartilage tragal doublé de son périchondre (2\%) (TableaulI).

\begin{tabular}{|c|c|c|c|c|}
\hline \multicolumn{2}{|c|}{ Résultat anatomique } & Guérison & Récidive & Perforation \\
\hline \multicolumn{2}{|c|}{ Résultat global } & $88 \%$ & $10 \%$ & $12 \%$ \\
\hline \multicolumn{2}{|c|}{ Aponévrose } & $73 \%$ & $15 \%$ & $12 \%$ \\
\hline \multicolumn{2}{|c|}{ Greffons cartilagineux } & $98 \%$ & $02 \%$ & 0 \\
\hline \multicolumn{2}{|c|}{ Périchondre } & $96 \%$ & $04 \%$ & 0 \\
\hline \multirow{3}{*}{ PDR } & Postérieures & $90 \%$ & $10 \%$ & - \\
\hline & Atticales & $93 \%$ & $7 \%$ & - \\
\hline & Globales & $95 \%$ & $5 \%$ & - \\
\hline
\end{tabular}

\section{Tableau II : Résultats anatomiques}

Les perforations secondaires sont exclusivement secondaires à une chirurgie de renforcement par greffe d'aponévrose.

Nous avons pu bénéficié d'un suivi audiométrique supérieur à 1 an chez tous nos patients. Nous avons calculé le rinne résiduel $(\mathrm{RR})$, c'est-à-dire la différence entre le rinne 
préopératoire et le rinne postopératoire. Les résultats auditifs ont été considérés satisfaisants au dessus de $20 \mathrm{~dB}$ de $\mathrm{RR}$, entre 20 et $30 \mathrm{~dB}$ ils sont été considérés comme moyens et au-delà de $30 \mathrm{~dB}$ les résultats sont mauvais.

L'analyse des données audiométriques montre que le résultat fonctionnel est globalement bon. En effet, 40 patients ont vu leur audition s'améliorer et 62 ont gardé une audition inchangée.

L'analyse des résultats fonctionnels en fonction du siège des poches de rétraction montre que la fermeture complète du Rinne a concerné $16 \%$ des poches de rétraction toutes localisations confondues. Ce pourcentage atteint $45 \%$ pour les poches postérieures et $60 \%$ pour les rétractions globales.

L'étude de l'audition postopératoire en fonction du stade évolutif de la poche montre que les plus mauvais résultats sont obtenus pour les poches de stade III. L'analyse des résultats fonctionnels en fonction des matériaux de renforcement révèle que les meilleurs résultats auditifs sont obtenus chez les patients ayant bénéficié d'un greffon chondropérichondral (43\% de gain auditif) (Tableau III).

\begin{tabular}{|c|c|c|c|c|c|c|c|}
\hline \multicolumn{2}{|c|}{ Résultats auditifs } & \multicolumn{2}{|c|}{ Inchangé } & \multicolumn{2}{|c|}{ Gain } & \multicolumn{2}{|c|}{ Aggravation } \\
\hline & & $\mathrm{n}$ & $\%$ & $\mathrm{n}$ & $\%$ & $\mathrm{n}$ & $\%$ \\
\hline \multicolumn{2}{|c|}{ Taux global } & 62 & 46 & 40 & 29 & 32 & 23 \\
\hline Stade & Stade III & 70 & 62 & 30 & 26 & 11 & 9 \\
\hline \multirow[t]{3}{*}{ Matériaux } & Aponévrose & \multicolumn{2}{|c|}{$58 \%$} & \multicolumn{2}{|c|}{$20 \%$} & \multicolumn{2}{|c|}{$22 \%$} \\
\hline & Cartilage et périchondre & \multicolumn{2}{|c|}{$46 \%$} & \multicolumn{2}{|c|}{$43 \%$} & \multicolumn{2}{|c|}{$11 \%$} \\
\hline & Périchondre & \multicolumn{2}{|c|}{$57 \%$} & \multicolumn{2}{|c|}{$22 \%$} & \multicolumn{2}{|c|}{$21 \%$} \\
\hline \multirow[t]{3}{*}{ Siège } & PDR Postérieure & \multicolumn{2}{|c|}{$45 \%$} & \multicolumn{2}{|c|}{$28 \%$} & \multicolumn{2}{|c|}{$27 \%$} \\
\hline & PDR Globale & \multicolumn{2}{|c|}{$60 \%$} & \multicolumn{2}{|c|}{$25 \%$} & \multicolumn{2}{|c|}{$15 \%$} \\
\hline & PDR Atticale & \multicolumn{2}{|c|}{$40 \%$} & \multicolumn{2}{|c|}{$35 \%$} & \multicolumn{2}{|c|}{$25 \%$} \\
\hline
\end{tabular}

Tableau III : Résultats fonctionnels

\section{DISCUSSION}

Les poches de rétraction constituent une entité clinique particulière. Elle reste un sujet d'actualité en otologie. En effet, elles n'ont cessé de susciter des discussions et des controverses depuis longue date tant par leur étiopathogénie par leur histoire clinique et leur génie évolutif [1]. En effet, la filiation entre otite séromuqueuse, les poches de rétraction et le cholestéatome a été pressentie depuis longtemps [1].

Le facteur essentiel à l'origine des poches de rétraction est l'existence d'un processus inflammatoire chronique au niveau des cavités de l'oreille moyenne et de la muqueuse tubotympanique [1, 2].

Faire le bilan lésionnel et classer la poche sont nécessaires pour la prise en charge ultérieure. Une fois la poche constituée, l'évolution naturelle vers la maladie cholestéatomateuse est imprévisible. Pour Gersdorff, 3\% des poches évolueront vers le cholestestome dans les 4 ans qui suivent la découverte clinique de la rétraction tympanique [3, 4].

Plusieurs classifications sont proposées, celle de Charachon demeure la plus classique et la plus utilisée bien qu'elle ne tienne pas compte du siège et de la marginalité de la poche $[5,6]$.

Le traitement des poches de rétraction tympanique dépend essentiellement du caractère fixé ou non $[7,8,9$, 10].

Les poches mobiles posent le moins de problème, la surveillance périodique serait suffisante. Si la poche s'étend sans perdre pour autant son caractère mobile, l'aérosolothérapie sonique peut trouver alors son indication. Un aérateur transtympanique peut être placé en cas d'otite séromuqueuse surajoutée [11, 12].

Il en est de toute autre attitude pour les poches fixées. En effet, elles nécessitent dans la majorité des cas le recours à la chirurgie pour éviter les lyses ossiculaires et surtout l'évolution vers la maladie cholestéatomateuse [12].

Pour les poches contrôlables, l'indication opératoire doit être modérée en fonction de la gêne sociale du patient et la survenue d'épisodes otitiques de surinfection [13]. Dans notre série, nous avons opérés 20 PDR de stade II. Pour les poches de rétraction de stade III, l'indication opératoire est formelle selon la plupart des auteurs [13, 14, 15]. Dans ces cas, le traitement chirurgical comporte trois volets : l'ablation de la poche, la vérification de la perméabilité antroatriale, la reconstitution du cadre et la renforcement tympanique.

Dans les cas où la poche adhère intimement aux osselets ou aux différents éléments de la gouttière postérieure, une tympanotomie postérieure permettra de contrôler toute la région du rétrotympanum.

Après l'exérèse de la poche, la majorité des auteurs ont recours actuellement au greffon chondropérichondral à partir du cartilage tragien pour la reconstitution du cadre et le renforcement tympanique [15].

II s'agit d'un matériel rigide qui confère à la greffe tympanique une solidité évitant les récidives de rétraction. Dans notre série, le taux d'échec anatomique sur greffon chondropérichondral est de $14 \%$. II serait du à la persistance du dysfonctionnement tubaire, d'ailleurs, la rétraction se produit presque toujours en dehors de la zone tympanique armée de cartilage.

Sur le plan fonctionnel, nous avons pu obtenir de bons résultats auditifs avec le greffon de cartilage tragal en réalisant une stapédomyringopexie cartilagineuse (37 cas) et une ossiculoplastie par une prothèse d'hydroxyapatite (9 cas). En effet, le cartilage a l'avantage de ne pas se solidariser au cadre osseux ou à la néocollumelle. La mobilité tympanique et la transmission sont ainsi préservées. Cependant, on reproche au greffon chondropérichondral son opacité ce qui empêcherait de détecter lors des 
contrôles otoscopiques postopératoires, une éventuelle récidive choléstéatomateuse $[16,17,18]$. Pour cela, un 2ème temps est indispensable au bout de un an, pour vérifier l'absence de résidu épidermique [19, 20, 21]. Le scanner peut aider en préopératoire avant le second look en apportant des éléments prédictifs de récidive.

\section{CONCLUSION}

La chirurgie de renforcement tympanique dans les poches de rétractions a pour but de s'opposer à l'atelectasie et à la progression évolutive incontestable vers l'oti- te chronique cholestéatomateuse.

Le greffon chondropérichondral constitue un bon moyen pour renforcer la membrane tympanique et éviter les récidives de rétraction sans compromettre l'état auditif surtout à un stade évolutif précoce.

\section{REFERENCES}

1- Roulleau P, Martin Ch. Poches de rétraction et états pré-cholestéatomateux. Les monographies du cca Wagram $n^{\circ} 26$.

2- Deguine Ch. Pathogénèse du cholestéatome. Rev Laryngol $1995 ; 1$ : 61-63. $3-$ Tos M. Upon the relation ship between secretary sequelae in adults. J. Laryngol Otol $1981 ; 95$ : 1011-22.

4- Gersdorff M., Cornelis J. Oreille controlatérale en cas de cholestéatome. Rev Laryngol $1992 ; 1: 35$

5- Martin Ch., Martin H., Prades J., Marzougui N., Chelikh L. Intérêt de la chirurgie de renforcement de tympan dans les poches de rétraction tympaniques. J. F. d'ORL $1997 ; 46: 91-4$

6- Charachon R. Classification des poches de rétraction. Rev Laryngol $1988 ; 3$ : 205-7.

7- Wapsh R, Pracy J, Harding L. Bowdier a management of retraction pockets of the pars tense in children. J. Laryngo Otol $1995 ; 109: 817-20$.

8- Tay H, Mills R, Phil M. Tympanic membrane atelectasis in childhood otis media with effusion. J. Laryngol Otol $1995 ; 109: 495-8$.

9- Roger G, Tashijan G, Roelly P, Rahmi H, Lacombe H, Garabedian E. Poches de rétractions fixées et le cholestéatome de l'enfant. Ann Oto Laryngol 1994 ; $111: 103-9$

10- Dubreuil Ch. Les poches de rétraction. J. F. d'ORL $1989 ; 9$ : 422-6.

Chiossone E. Preventive tympanoplasty in children. Rev Laryngol 1995 ; 2 :

137-139.
11- Ars B, Piret N. Rétractions de la membrane tympanique. Cahiers d'ORL $1993 ; 3: 89-93$

12- Roulleau P. Les poches de rétractions fixées et leur traitement. Rev Laryngol 1988 ; 3. 209-11.

13- Charachon $R$, Lavieille J, Boulat $E$, Verdier $N$. Le traitement chirurgical des poches de rétraction. Rev. Soc. Fr. ORL, $1996 ; 36: 11-8$.

Dornhoffer J. Hearing results with cartilage tympanoplasty. Laryngoscope 1997; $107: 1094-9$.

14- Harner S. Management of posterior tympanic membrane retraction. Laryngoscope $1995 ; 105: 326-329$

15- Florent A, Trang M, Jaulin J, Baril C, Roulleau P. Poches de rétraction tympaniques à propos de 106 cas. Ann Oto Laryngol 1987 ; 104 : 519-33.

16- Uziel A, Giner R. Traitement chirurgical des poches de rétraction tympaniques. J. F. d'ORL $1989 ; 38: 427-30$.

17- Martin Ch, Prades J, Calloch F, Mayaud R. Endoscopie et chirurgie des poches de rétraction tympaniques. Cah. d'ORL $1995 ; 2$ : 85-92.

18- Yung M W. Retaction of the pars tensa, long term results of surgical trearement 1997 ; 22: 323-6.

19- Belkadhi M. Traitement chirurgical des poches de rétraction tympanique J.STORL 1999, 2 : 8-11. 